\title{
CADENAS GLOBALES DE VALOR: UNA REVISIÓN BIBLIOGRÁFICA*
}

\author{
Recibido: 11 de enero de 2019 - Aprobado: 15 de abril de 2019 \\ https://doi.org/10.22395/seec.v22n51a4 \\ Javier Pérez lbáñez ${ }^{* *}$
}

\section{RESUMEN}

Durante los últimos treinta años, la producción de bienes y servicios ha sufrido un proceso de deslocalización y fragmentación a nivel mundial. Esta nueva fase de la organización productiva recibió el nombre de cadenas globales de valor. Los estudios enmarcados en este entramado teórico se focalizaron en el desarrollo económico (Upgrading) y el gobierno de estas cadenas (gobernanza). El objetivo de este artículo, es sistematizar cómo han sido estudiadas las cadenas globales de valor, para identificar los potenciales aportes que esta teoría puede hacer al entendimiento de esta nueva fase del desarrollo productivo. Para cumplir dicho propósito, se analizaron publicaciones académicas, de instituciones públicas y centros de estudios. Como resultado se obtuvo un profundo estado del arte sobre la temática.

\section{PALABRAS CLAVE}

Cadenas globales de valor; desarrollo económico; gobernanza; upgrading.

\section{CLASIFICACIÓN JEL}

F13, L22, 024, F63

\section{CONTENIDO}

Introducción; 1. Gobiernos de las cadenas; 2. Desarrollo y cadenas; 3 . Reflexiones finales; Bibliografía

Este artículo es un breve resumen de los principales aportes existentes a la fecha sobre la temática cadenas globales de valor. El mismo es el resultado de una extensa revisión bibliográfica realizada por el autor en el marco de su tesis doctoral. El autor posee una beca doctoral financiada por el Consejo Nacional de Investigaciones Científicas y Técnicas (Conicet).

* $\quad$ Licenciado en Sociología, Universidad de Buenos Aires, Buenos Aires, Argentina. Jefe de trabajos prácticos en la Universidad Nacional de Avellaneda, Buenos Aires, Argentina. Profesor asociado Universidad Nacional de San Martín, Buenos Aires, Argentina. Correo electrónico: javier.p.ibanez@gmail.com. Orcid: https://orcid. org/0000-0002-8097-4504 


\title{
GLOBAL VALUE CHAINS: A BIBLIOGRAPHICAL REVIEW
}

\begin{abstract}
During the last thirty years, production of goods and services has undergone a relocation and fragmentation process worldwide. This new phase of productive organization was called global value chains. The studies framed in this theoretical framework focused on economic development (Upgrading) and the government of these chains (governance). The objective of this article is to systematize how global value chains have been studied to identify potential contributions of such theory to the understanding of this new phase of productive development. To achieve this purpose, academic publications from public institutions and study centers were analyzed. As a result, a deep state of the art on the subject was obtained.
\end{abstract}

\section{KEYWORDS}

Global value chains; economic development; governance; upgrading

\section{JEL CLASSIFICATION}

F13, L22, 024, F63

\section{CONTENTS}

Introduction; 1. Chain governments; 2. Development and chains; 3. Final thoughts; Bibliography

\section{CADEIAS GLOBAIS DE VALOR: UMA REVISÃO BIBLIOGRÁFICA}

\section{RESUMO}

Durante os últimos trinta anos, a produção de bens e serviços tem sofrido um processo de deslocalização e fragmentação em nível mundial. Essa nova fase da organização produtiva recebeu o nome de "cadeias globais de valor". Os estudos delimitados nesse contexto teórico estão focados no desenvolvimento econômico (upgrading) e no governo dessas cadeias (governança). O objetivo deste artigo é sistematizar como as cadeias globais de valor vêm sendo estudadas para identificar as potenciais contribuições que essa teoria pode fazer ao entendimento dessa nova fase do desenvolvimento produtivo. Para cumprir esse propósito, foram analisadas publicações acadêmicas de instituições públicas e de centros de estudos. Como resultado, foi obtido um profundo estado da arte sobre a temática.

\section{PALAVRAS-CHAVE}

Cadeias globais de valor; desenvolvimento econômico; governança; upgrading.

\section{CLASSIFICAÇÃO JEL}

F13, L22, 024, F63

\section{CONTEÚDO}

Introdução; 1. Governos das cadeias; 2. Desenvolvimento e cadeias; 3. Reflexões finais; Bibliografia 


\section{INTRODUCCIÓN}

La fragmentación y deslocalización de la producción ha sido una de las graves transformaciones ocurridas en la económica mundial de los últimos 30 años. A partir de la década del ochenta, las empresas comenzaron a deslocalizar sus procesos productivos a nivel mundial. Como consecuencia, el valor comenzó a ser agregado en múltiples países antes de llegar a los consumidores finales. Según Gereffi y Sturgeon (2013, p.329) este proceso fue posible gracias a la caída de las barreras arancelarias, el afianzamiento a nivel mundial de la Organización Mundial del Comercio (OMC) como órgano regulador de los intercambios globales ${ }^{1}$ y las políticas asociadas al consenso de Washington.

Para dar cuenta de este nuevo fenómeno, surge el concepto ${ }^{2}$ de cadenas globales de valor (CGV). La concepción de cadena de valor, fue utilizada para analizar la secuencia de actividades necesarias en la manufacturación de un determinado producto, no solo su parte tangible, sino también de toda una gama de servicios asociados (comercialización, distribución, postventa, etc.). Lo novedoso surge de la dimensión global que tomó este fenómeno, al dividirse la producción en múltiples países y al ocuparse cada uno de ellos de una parte diferente en la agregación de valor. Los estudios que utilizan las CGV como marco teórico pretenden explicar el proceso por el cual la producción se desintegró a través de la integración del comercio internacional (Feenstra, 1998, p. 31) al crearse una compleja red entre proveedores y casas matrices.

En el núcleo de este cambio mundial, se encuentra la decisión de las compañías multinacionales de focalizar el núcleo de sus negocios en la innovación, el marketing y los segmentos que más valor agregan, dejando de lado la propiedad directa de los procesos productivos más genéricos y centrados en el volumen. El enfoque de las CGV resalta que la coordinación y el control a escala global de la producción pueden lograrse sin la propiedad directa de los recursos en otros países.

Esto representa un quiebre con las estrategias de integración vertical, típica del modelo fordista, dado que se vuelve más beneficioso para las corporaciones multinacionales deslocalizar (offshoring) y externalizar (outsourcing) parte de su proceso de producción (Gereffi, Humphrey y Sturgeon, 2005; Porta, Santarcángelo y Schteingart, 2017; Milberg y Winkler 2013), antes que concentrar la propiedad del mismo a nivel local, lo que se hace es transferir actividades consideradas como no

\footnotetext{
1 La incorporación de China (2001) y Rusia (2012) a la Organización Mundial del Comercio (OMC) marcan el punto a partir del cual se hacen globales las reglas impuestas por esta institución.

2 Este artículo pretende abordar la dimensión teórica de a las CGV. Este recorte no excluye que también pudiese existir una revisión similar en términos metodológicos o técnicos. Para una búsqueda en tal sentido se recomienda la recopilación de Dussel (2018).
} 
esenciales a empresas de otro país, las cuales pueden residir en el exterior o no. En suma, las empresas multinacionales vieron reforzado su rol en la economía mundial de la mano de la profundización de la CGV.

El eje que organiza los nuevos arreglos productivos es la búsqueda de ventajas económicas por parte de las empresas multinacionales. Cardeillac (2013, p. 7) sostiene que la estrategia es quebrar el proceso productivo en varios segmentos discretos y con formas específicas de organización. Frente al lento crecimiento económico en los países centrales, las empresas multinacionales se establecieron en las naciones en desarrollo para aprovechar los bajos costos productivos y laborales y exportar de vuelta a sus economías de origen o abastecer estos mismos mercados. Estos países emergentes, ofrecían grandes cantidades de trabajadores a bajo costo, capacidades productivas e infraestructuras en crecimiento, abundantes materias primas y también la posibilidad de vender en un nuevo mercado doméstico que progresaba al ritmo del surgimiento de nuevas clases medias (Gereffi y Sturgeon, 2013).

Ahora bien, según Milberg y Winkler (2013, p. 239) estos cambios en las estrategias empresariales deben ser analizados en el contexto de un mundo en el que ocurrieron grandes transformaciones que determinaron dichas estrategias. Primero, la aparición de desarrollos tecnológicos en comunicaciones, que permiten controlar la producción a grandes distancias o transportar mercancías a menor costo y más rápido de un continente a otro. Segundo, la integración de Brasil, Rusia, India y China al comercio mundial como economías de mercado, generó nuevos destinos donde colocar producciones como así también abundante mano de obra dispuesta a trabajar por menores salarios que en los países desarrollados. Tercero, el reemplazo de la sustitución de importaciones por el desarrollo basado en las exportaciones como eje central de las políticas industriales de los países en desarrollo.

La academia ha realizado grandes esfuerzos teóricos para elaborar un entramado conceptual que pueda dar cuenta de este nuevo fenómeno, al constatar la capacidad explicativa de estas teorías con la multiplicidad de diferentes cadenas existentes en cada rama productiva. De manera constante se actualizan las ideas con nuevos aportes para dar cuentas de nuevas dimensiones y procesos particulares. Las CGV se constituyen como un marco teórico todavía en creación que se nutre de los diferentes conceptos de distintas disciplinas, tales como la economía política, la geografía económica, los estudios sobre el desarrollo, la administración de empresas, la ciencia política, la economía internacional y la sociología económica.

En este sentido, existen discusiones abiertas entre los diferentes investigadores en torno a dos ejes centrales que constituyen esta teoría. Este artículo se estructura en diferentes capítulos que pretenden ser un primer abordaje a estos debates. En el 
primer apartado, se consideran las formas específicas bajo las cuales se organizan estas cadenas. En el último, se revisan los impactos de las mismas en los procesos de desarrollo económico.

\section{GOBIERNO DE LAS CADENAS}

Con la generalización del concepto de CGV, la academia comenzó a centrarse en el análisis de la gobernanza, es decir, cómo se organiza y quién lidera esta nueva fase productiva mundial. Gibbon, Bair y Ponte (2008, p. 4) sostienen que el concepto permite resaltar las prácticas concretas y formas organizacionales particulares bajo las cuales las empresas líderes y otros actores, en el contexto de un particular división internacional de trabajo, toman decisiones. Dado que el centro explicativo está depositado en la agencia de los actores, el concepto de gobernanza aleja a la teoría de las CGV de otros abordajes de las temáticas, en las que prima una visión más estructuralista en torno a la agencia de los actores. La globalización, más que una fase del capitalismo mundial, pasa a ser consecuencia de las decisiones concretas tomadas por actores concretos, normalmente grandes empresas multinacionales, en circunstancias concretas.

El primer antecedente puede encontrarse en la particular recuperación que realiza Gereffi (1994) del concepto de cadenas globales de mercancías (GCC por su sigla en inglés) elaborado por Hopkins y Wallerstein (1986). Esta noción en sus orígenes era utilizada para dar cuenta del proceso por el cual los Estados nacionales tenían la capacidad de moldear el comercio internacional a través de herramientas como tarifas o regulaciones al contenido importado de los productos. Gereffi (1994) invierte el orden explicativo y focaliza sobre la acción de las empresas y la capacidad de las mismas de limitar el poder estatal en el contexto de la apertura económica y la liberalización comercial de fines de la década de los 1980. Con este cambio de objeto de estudio, del Estado-nación al de acción empresaria, emerge la pregunta sobre cómo las firmas organizan la producción a nivel mundial y surge la idea de gobernanza.

Como mencionamos al principio de este apartado, el concepto de gobernanza surge para explicar las distintas formas bajo las cuales las empresas organizan su producción transfronteriza. En la concepción original de Gereffi (1994), se desprendían dos tipos ideales de gobernanza en función del rol que ocupasen las empresas líderes en las cadenas, conducidas por productores (producer-driven) o por compradores (6uyer-driven). Gibbon et al. (2008, pp. 5 y 16), Ponte y Gibbon (2005, p. 6) y Sturgeon (2008, p. 10) proponen denominar esta concepción de gobernanza como conducción. 
Los primeros postulados de Gereffi (1994) sostienen que, cuando una cadena es conducida por proveedores, los intercambios se circunscriben a las redes que generan de manera interna o externa grandes empresas manufactureras a la hora de abastecer su producción. El nombre de esta tipología de cadena está dado por el liderazgo que establecen las empresas productoras sobre el resto de los actores. En este tipo de cadenas se inscriben la industria automotriz, la aviación, la computación o la producción de maquinarias. Son intensivas en capital, lo cual genera grandes barreras a la entrada de nuevos competidores. Lo más distintivo es el fuerte control generado desde las casas matrices sobre el proceso productivo y los proveedores. En cambio, las cadenas conducidas por compradores están dominadas por grandes vendedores minoristas (retailers) y compañías con marcas instaladas que desempeñan el rol de pívot entre muchos pequeños proveedores a nivel mundial y los consumidores. Estos grandes compradores, dado el gran volumen de compra que manejan, tienen el poder de moldear a todos los actores de la cadena sin poseer en propiedad directa una sola factoría. Suelen ser ramas pocos sofisticadas e intensivas en mano de obra. Santarcángelo, Schteingart y Porta (2017, p. 108) mencionan que un ejemplo clásico de este tipo de cadenas es la industria indumentaria, del juguete o del calzado

Gibbon et al. (2008, pp. 8 y 20), Ponte y Gibbon (2005, p. 9) y Sturgeon (2008, p. 14), al historizar la evolución conceptual del entendimiento de las cadenas como conducción argumentan que, si bien en un comienzo esta tipología tuvo una gran acogida por parte de la academia dado su gran poder explicativo sobre los actores de la cadena, sus relaciones, interdependencias y disputas de poder, en el corto plazo debió ser revisada al volverse rígida y estática. La crítica estuvo centrada en el excesivo empirismo con el cual fueron elaboradas las categorías originales dado que muchos estudios encontraron dificultades para encasillar sus casos dentro de las mismas. A su vez, la generalización del outsourcing como estrategia empresarial hacia fines de la década de 1990 generó que muchas cadenas conducidas por productores comenzaran a actuar como conducidas por compradores y que, por tanto, perdiese sentido la división original.

Frente a estos desafíos Sturgeon (2008, p. 4) relata que un grupo de académicos comienza a entablar reuniones a principio de siglo XXI con el objetivo de elaborar un marco teórico que pudiese captar mejor los cambios acontecidos en el comercio internacional. En estas reuniones se utiliza por primera vez el concepto de CGV y se propone avanzar hacia una teoría de la gobernanza basada en las redes productivas en el que se focaliza un carácter más relacional de los intercambios. 
Gereffi et al. (2005) en su artículo más conocido³, logran resumir estas discusiones a partir de construir una tipología de la organización de las cadenas de valor basada en tres variables, la complejidad de la venta, la capacidad de codificar la información incluida en la misma y los desarrollos técnicos de la base de proveedores. Esta concepción de gobernanza fue denominada de forma posterior por Gibbon, Bair y Ponte (2008, pp. 14 y 21) y por Ponte y Gibbon $(2005$, p. 12) como coordinación, dado que cambia el foco de toda la cadena hacia las transacciones interempresa.

En esta nueva conceptualización una empresa agrega complejidad a la cadena en la medida en que incorpora nuevas demandas a los proveedores o busca aumentar la diferenciación o especificidad de los productos que desean comprar. Por el contrario, la estandarización de los productos y los procesos reduce la complejidad. En cuanto a la información, su variación es similar en la medida en que puede codificarse. Por ejemplo, a través de la adopción de técnicas estandarizadas se reduce la complejidad trasmitiéndose de manera eficiente. Por último, una cadena puede variar en función de las capacidades técnicas que tengan los proveedores de dar cuenta de los requerimientos técnicos y de la calidad demandados por los consumidores.

Detrás de estas variables tiene un lugar central la teoría de los costos de transacción de activos específicos. La misma sostiene que un activo específico es aquel cuyo valor depende de manera sustancial de la continuidad contractual, de manera tal que la ruptura del vínculo genera costos para ambas partes. Porta et al. (2017, p.2) mencionan como ejemplo de estos activos específicos las capacidades tecnológicas, innovadoras, comercializadoras, financieras o el mismo desarrollo de la marca. De esta manera, los contratos ordenan la tensión que genera el lidiar con los activos específicos en el marco de una relación comercial. Cardeillac (2013, p.11) menciona que, con anterioridad al proceso de globalización de la producción, las empresas tendían a la integración vertical para evitar el costo derivado de romper relaciones contractuales con sus proveedores.

Ahora bien, la teoría de los activos específicos, elaborada por Williamson, sostiene que, a lo largo de la relación contractual, se generan oportunidades de las cuales las dos partes involucradas podrían sacar provecho sobre la otra al romper la relación comercial y capitalizar los activos específicos (Sturgeon 2008, p. 16). Los aportes de la sociología económica de Granovetter (1985), al sostener que la acción económica está enraizada socialmente, resaltan que la confianza tiende a surgir como forma de organizar la relaciones entre firmas y que, por tanto, es posible sostener complejos intercambios en el tiempo. Este enfoque de las CGV,

Nos referimos a La gobernanza de las cadenas globales de valor. Este artículo fue citado más de cinco mil veces según Google Académico. 
permite dar cuenta de cómo son manejados los activos específicos en el proceso de deslocalizar o externalizar algún proceso productivo. Así, quedan conformados cinco tipos analíticos de formas que asume la gobernanza en las CGV en función de las variables antes descriptas:

A. Mercados. Los vínculos que se generan en este caso están organizados a través de los mercados y el precio. De esta manera, si bien los intercambios se sostienen en el tiempo, el costo de cambiar de socios es bajo tanto para el comprador como para el proveedor. En este tipo de cadenas, la información es codificada de manera simple, la especificación de los productos es baja y los proveedores tienen la capacidad de fabricar los productos con poca injerencia de los consumidores. La coordinación es muy poca y lo compradores responden al nivel de precios a la hora de decidir una compra.

B. Cadenas de valor modulares. En este tipo particular de relación, los proveedores arman productos de acuerdo a las demandas específicas de los clientes. Si bien se ofrece un producto terminado en su totalidad, son los proveedores los que se hacen cargo de la tecnología incorporada en el proceso productivo, lo cual disminuye la capacidad de ofrecer productos específicos, y realizan las inversiones necesarias de capital y materia prima. Se extiende la codificación de la información de tal manera que se permite sostener mayores niveles de complejidad de los productos con poca supervisión de los compradores. Esto se logra también a partir de reducir la variación de productos fabricados y al unificar componentes, productos y procesos. En este caso, la clave está dada por la capacidad de codificar la información en las transacciones, por lo que se conservan varias de las ventajas de las cadenas de mercado, como la velocidad, la flexibilidad y los bajos costos implicados en el cambio de socios.

C. Cadenas de valor relacionales. Aquí se generan complejas interacciones con altos grados de dependencia entre compradores y proveedores. Como contrapartida, se logran grandes niveles de especificidad en los activos generados. En este esquema, la confianza basada en la reputación es central. Este es un caso en el que no se puede codificar la información, las ventas son complejas y la capacidad de los proveedores es alta. Se requieren altos grados de coordinación e incluso interacciones cara a cara, lo que supone grandes costos al cambiar de socios y, por tanto, es esperable la existencia de multas a aquellos que rompan los contratos.

D. Cadenas de valor cautivas. En este caso, pequeños productores dependen de los intercambios con compradores más grandes, lo que supone que los costos de 
romper la asociación sean más altos para los primeros que para los segundos. Estas cadenas implican un gran control y monitoreo por parte de las empresas líderes, debido a que la complejidad es alta, la capacidad de codificar información es baja y, a diferencia de la anterior forma, la capacidad de los proveedores es baja. Las empresas líderes intervienen y controlan a sus proveedores de manera cercana, y aumenta, en consecuencia, la dependencia internacional. El objetivo de las empresas líderes, es que los proveedores no se diversifiquen hacia otros clientes, de ahí el nombre de cautivo.

E. Cadenas de valor jerárquicas. Esta forma de gobernanza se caracteriza por la integración vertical, se ejerce el control desde las sedes centrales hacia las subsidiarias. Aquí no son posibles ninguna de las tres variables, la información es imposible de codificar, los intercambios son muy complejos y no existen proveedores capaces de responder a las necesidades de las empresas líderes. Por tanto, las empresas se ven obligadas a producir ellas mismas productos que ningún otro actor está en condiciones de hacer.

Estas cinco formas de gobernanza, lejos de ser una teoría sobre la globalización, son presentadas como tipos ideales a partir de los cuales entender cómo diferentes estadios en la agregación de valor se enlazan y posibilitan predecir los efectos que tendrían sobre la misma alguna modificación en las tres variables propuestas.

Por último, la gobernanza como normatividad o normalización surge como una tercera forma de entender el gobierno de las CGV a partir de un conjunto de críticas que suscitó el entendimiento de las cadenas como coordinación. Las divergencias se estructuran en torno al excesivo peso explicativo depositado en la interrelación entre actores y el consecuente desdibujamiento sobre la cadena que se genera en su conjunto (Gibbon et al., 2008, p. 9).

Ponte y Gibbon (2005, p. 9) ponen en el centro explicativo de la gobernanza como normalización a la teoría de las convenciones y el rol que cumple la calidad en los intercambios internacionales. A partir de estos elementos se redefine la gobernanza como la capacidad de las empresas líderes de determinar los estándares de calidad de los productos que se comercian, incluso generando disputa entre actores por definir y redefinir estos patrones.

La teoría de las convenciones establece las formas sociales bajo las cuales se crean estos parámetros de calidad al focalizarse en los aspectos intersubjetivos y normativos. Las convenciones son un conjunto de expectativas de acción mutuas que incluye las instituciones, pero no las agota. Estas afectan las formas en las que los agentes económicos moldean sus interpretaciones sobre la calidad de los 
productos y generan guías para la acción con base en estos estándares socialmente construidos (Gibbon et al., 2008; Velázquez y Tenorio, 2017). En síntesis, Ponte y Gibbon (2005, p. 10) proponen entender las convenciones como un sistema de expectativas recíprocas sobre el compartimiento de otros. De esta manera se genera un contexto normativo, de aquí el nombre del tipo de gobernanza, bajo el cual se condicionan las expectativas de los actores de las cadenas y se determinan las cualidades requeridas de los bienes y servicios para poder ser comerciados.

Las convenciones pueden estar institucionalizadas, como así también emerger en el proceso mismo de la interacción entre empresas (Gibbon et al., 2008; Ponte y Gibbon, 2005). Como resultado, en la definición de la calidad intervienen actores externos a las cadenas, instituciones u organizaciones que poseen la legitimidad social para establecer los parámetros de calidad de los productos, como así también los consumidores y sus pautas de consumo estructuradas en estas convenciones (Velázquez y Tenorio, 2017) ${ }^{4}$. A su vez, se retorna a una concepción en la que existen empresas líderes y por tanto conducción en la cadena, disputa y diferencias de intereses. Así, si bien existe una tendencia hacia un mundo más global o de redes, en las cadenas no desaparece la conducción, sino que es ejercida de otra manera (Ponte y Gibbon, 2005, 2008).

Bajo esta definición las coordinaciones pueden ser múltiples, incluso dentro de una misma cadena, pero la gobernanza una sola. Ponte y Gibbon $(2005 ; 2008)$ proponen cuatro tipos de coordinaciones basadas en convenciones sobre la calidad: i) mercantiles, definidas por el valor del mercado y en las que la diferencia del precio es explicada por la calidad del producto; ii) domésticas, en donde la incertidumbre sobre la calidad de los productos se resuelve a través de la confianza gestada en relaciones de largo plazo y el conocimiento de marcas determinadas; iii) industriales, explicadas por la eficiencia productiva y en las que los patrones de calidad son puestos por actores externos que determinan normas y estándares sobre los productos; iv) cívicas, influidas por el bienestar colectivo, social o ambiental (Ponte y Gibbon, 2005; Velázquez y Tenorio, 2017).Estos cuatro posibles tipos de coordinaciones pueden darse a lo largo de toda la cadena, y coexistir a su vez con formas bastante homogéneas de gobernanza. Para la definición de esta última, los autores vuelven a utilizar la concepción original de Gereffi (1994) de conducción por compradores o por productores.

\footnotetext{
${ }^{4}$ Existe otra dimensión en la relación entre convenciones y CGV que no se incluirá por cuestiones de espacio. La misma establece relaciones directas entre las formas legítimas de organización empresarial y la coordinación hacia adentro de las empresas y los efectos que esto tiene sobre la organización de la producción (Ponte y Gibbon, 2005).
} 
En resumen, la gobernanza como normatividad resalta los aspectos normativos por los cuales los actores en una cadena construyen concepciones sobre la calidad de los productos intercambiados y disponen acciones y expectativas de acción como consecuencia de este proceso. Se focaliza en la cadena en un sentido más amplio, al incluir más actores sociales, como las instituciones, los consumidores y la definición de la gobernanza de los involucrados en los procesos productivos. Por último, se retoma una concepción de disputa de intereses y litigio dentro de la cadena, lo que se aleja de la concepción un poco más armónica y de consenso planteada hasta el momento.

Esta última concepción analizada continúa siendo minoritaria frente al gran conceso alcanzado por la propuesta de Gereffi et al., (2005). Por tanto, el entendimiento de la gobernanza como coordinación sigue siendo hegemónico en la academia a la hora de abordar los estudios sobre las CGV.

\section{DESARROLLO Y CADENAS}

De igual forma, cabe analizar el rol de las cadenas de valor en los procesos de desarrollo económico. Es posible identificar al menos tres posturas. Primero, aquellas que utilizan este marco conceptual para sustentar una agenda de reformas asociadas al libre comercio. Según Dalle, Fossati y Lavopa (2013, p. 5), en este primer agrupamiento se encuentran las posturas de los grandes organismos internacionales como la OMC y la Organización para la Cooperación y Desarrollo (OCDE). Segundo, los trabajos que sostienen que la integración a estas cadenas trae grandes ventajas en términos de innovación y crecimiento productivo, pero que este beneficio depende de la capacidad de intervención estatal en este proceso. Tercero, las posturas que tienen una visión menos optimista sobre el rol de las cadenas globales en el desarrollo económico basadas en las teorías de los sistemas innovadores.

La primera de estas posiciones sostiene que no todos los países están involucrados de igual manera en las CGV. Esto se debe a condiciones estáticas (como recursos naturales o localización geográfica), y a asuntos que pueden ser modificados a través de políticas de Estado (OMC, OCDE y Banco Mundial, 2014). Si los países quieren participar de la división de las riquezas originadas en las CGV, deben tener regímenes de comercio e inversión abiertos, predecibles y transparentes, dado que las medidas proteccionistas impactan negativamente sobre los proveedores internacionales, los inversores extranjeros y los productores domésticos (OMC, OCDE y Banco Mundial, 2013).

En distintos informes preparados para las reuniones del G-20, la OMC, la OCDE y el Banco Mundial $(2014 ; 2013)$ recomiendan a los países la apertura al comercio y 
a la inversión como políticas de reformas necesarias para el desarrollo económico. Bajo esta concepción, la eliminación de aranceles y el desmantelamiento de las barreras pararancelarias se vuelven medidas necesarias para reducir el costo de los negocios y de esta manera fomentar la mayor inserción en las CGV y, por tanto, el crecimiento económico.

En este sentido, son poco recomendables las políticas sectoriales que apunten a fortalecer determinadas ramas productivas por sobre otras dado que generan efectos distorsivos. Por el contrario, OMC, OCDE y Banco Mundial (2014, p. 8) entienden que son esperables las políticas económicas amplias tales como priorizar tanto las importaciones como las exportaciones, fomentar el comercio multilateral por sobre los acuerdos regionales, desestimar los esfuerzo en aumentar el contenido nacional de las importaciones y facilitar las importaciones de manera tal que el contenido tecnológico que les es propio se derrame hacia el resto de los actores, reducir los aranceles al comercio exterior y las medidas pararancelarias. En un informe del año anterior los mismos organismos internacionales (2013, p. 13) subrayan que, bajo esta concepción, existe un efecto distorsivo de los impuestos sobre el comercio, que es aún peor en la medida en que las exportaciones principales del país tengan un alto contenido importado.

Ahora bien, estos organismos internacionales reconocen que la apertura comercial puede conllevar la relocalización de recursos de actividades poco productivas a segmentos más eficientes. En esta transición, muchas personas pueden perder sus fuentes de trabajo y serán necesarias políticas sociales activas para facilitar el acceso a nuevas fuentes de trabajo a aquellos trabajadores desplazados.

La versatilidad y velocidad con la cual organismos como OMC, OCDE y Banco Mundial han logrado migrar sus concepciones sobre la relación entre comercio y desarrollo desde una visión más ortodoxa, asociadas a las clásicas ventajas comparativas, hacia incluir la teoría de las CGV en sus recomendaciones de política económica, pone un manto de sospecha sobre el particular abordaje que estos hacen del concepto. Al fin de cuentas, según lo analizado en los párrafos precedentes, se vuelve a caer en recomendaciones de corte generalístico que no distinguen segmentos, sectores, países, actores, lapsos de tiempo o condiciones estructurales particulares. En este sentido, el vínculo positivo que se le otorga desde estos organismos a la apertura comercial, la inversión extranjera, la reducción impositiva y la no intervención con el desarrollo económico en el marco de las cadenas globales dista mucho de la utilización original del concepto y de la realidad concreta bajo las cuales muchos países en vías de desarrollo han logrado mejorar su inserción internacional en este nuevo momento del comercio y la producción global. 
La segunda postura, está representada en los aportes que se han analizado hasta el momento, los cuales Dalle et al. (2013, p. 7) identifican como neoshumpeterianos. Autores como de Gereffi et al. (2005) sostienen que la mera apertura comercial no es garantía necesaria para asegurar el desarrollo económico. El concepto central que estructura su propuesta es el upgrading.

Se parte de reconocer que, el lugar que las empresas tengan en la CGV, puede determinar la incorporación a procesos productivos con alto valor agregado e intensivas en conocimiento, o quedar reducido a tareas de ensamblado intensivas en mano de obra. Esta división genera que no todos los países participen de manera igualitaria en los beneficios de la internalización. De hecho, se registra que las actividades que más agregan valor (diseño, investigación, desarrollo, marketing y distribución) quedaron asentadas en los países desarrollados, mientras que las que agregan poco valor se ubicaron en los países en desarrollo. El desafío, entonces, es lograr la participación en sectores de privilegio de la cadena que puedan generar un crecimiento sostenido en los niveles de ingreso. Esta tarea recibió el nombre de upgrading en la literatura especializada.

El aporte específico de las políticas activas en upgrading, es la creación de incentivos para que las empresas nacionales puedan escalar en la cadena global, desde los eslabones básicos hacia aquellos en los que se incorpora más valor. En un artículo clásico de Humphrey y Schmitz (2002, p. 6) se distinguen cuatro tipos de upgradings posibles: primero, con base en la mejora de los procesos de producción de manera tal de hacerlos más eficientes. Segundo, al crear bienes más sofisticados y con mayor tecnología. Tercero, desplazándose hacia otras actividades dentro de la cadena. Cuarto, intentar entrar en otras cadenas.

Es interesante rescatar que estos autores, a partir de sumar aspectos de la geografía económica y la teoría del cluster a las CGV, concluyen que los distintos tipos de gobernanza tienen consecuencias sobre las posibilidades de upgrading. De esta manera, las cadenas más cercanas a las jerárquicas generan mayores oportunidades para el rápido desarrollo de proveedores locales, pero los mismos, luego encuentran dificultades para escalar lugares en las cadenas. En síntesis, el proceso de desarrollo dentro de esta concepción implica moverse dentro de la cadena hacia eslabones superiores o hacia nuevas cadenas que agreguen más valor.

En términos de políticas públicas, el upgrading como propuesta dista mucho de la de industrialización por sustitución de importaciones (ISI) como eje del desarrollo. Una estrategia industrializadora dentro del CGV, debe contemplar de manera necesaria un primer momento de importaciones de partes y componentes que serán en una etapa posterior exportados en forma de productos finales. Dalle, 
Fossati y Lavopa (2013, p.7) sostienen que, bajo este conjunto de trabajo, es necesaria una fuerte intervención estatal que permita lograr el upgrading al reemplazar estos insumos importados y generar vínculos con los proveedores locales. De esta manera, la relación que la idea de upgrading tiene con la protección comercial resulta ambivalente. Por momentos, puede ser conveniente un cierto nivel de apertura hacia terceros países, pero en otros momentos resulta en extremo inconveniente para el desarrollo productivo.

Ahora bien, como carácter prevalente de los neoshumpeterianos, la inserción en las cadenas no es equivalente a desarrollo o upgrading. Estos procesos pueden registrar diferencias entre países e incluso dentro de distintas ramas, pero todos necesitan que el Estado delimite políticas industriales que logren generar condiciones para que las empresas escalen en la cadena.

A modo de cierre crítico, las propuestas del upgrading para este grupo de académicos pueden resultar bastantes distantes a las desarrolladas por los grandes organismos internacionales antes analizados. En particular, esta diferencia puede notarse en torno al rol del Estado, la asociación público-privada, los incentivos dirigidos hacia ramas o sectores particulares y la política industrial o sectorial. Ahora bien, en torno a las políticas comerciales no pareciese haber tanta diferencia. Si bien se reconoce que un cierto nivel de protección puede ser necesario, son fuertes las críticas hacia los modelos basados en la sustitución de importaciones o incluso hacia el regionalismo o los tratados bilaterales. Esta tención puede recogerse con mayor énfasis en los últimos artículos publicados por Gereffi (2018, p. 32).

Una tercera postura frente al problema del desarrollo y CGV se estructura desde la teoría de los sistemas de innovación (SI). Pietrobelli y Rabellotti (2011, p. 1261) sostienen que el enfoque de las CGV no aborda el problema de los mecanismos de aprendizaje de capacidades tecnológicas y de innovación en el marco de estas relaciones globales. Bajo su postulado, la inserción en cadenas globales posibilita en los países en desarrollo, no solo la oportunidad de insertarse en nuevos mercados, sino también la posibilidad de acceder nuevas tecnologías y conocimientos innovadores como eje central del upgrading.

La proximidad organizacional o relacional generada en los intercambios globales puede suplantar la importancia de la cercanía geográfica a la hora de propiciar, identificar y apropiar el flujo de conocimientos tácitos generados en las relaciones entre empresas. Aquí, cobran un rol central los sistemas de innovación nacionales (SIN), que deben abocarse a la absorción y adopción de tecnologías extranjeras ya existentes, más que buscar innovar en las fronteras tecnológicas. 
De esta manera, recuperan la idea de que las formas de gobierno en cada tipo de cadena tienen impactos sobre los mecanismos de aprendizaje, pero sostienen que los SIN aportan a reducir la complejidad de las transacciones y aumentar la capacidad de codificar la información, al posibilitar relaciones de mercado y debilitar las formas jerárquicas en las cadenas globales. Por tanto, la posibilidad de que las empresas nacionales queden encerradas en relaciones captivas disminuye en la medida que se fortalecen los SIN.

Desde una visión más pesimista Pessoa Matos, Cassiloato y Podcameni (2015) reconocen los aportes de la literatura de las CGV y de los SIN, pero sostienen que el upgrading como estrategia de desarrollo acarrea importantes errores conceptuales. Su crítica está dirigida a la visión simplista por la cual los lazos entre actores locales y empresas internacionales pueden llevar a procesos de aprendizaje innovadores. Si bien los aportes de la teoría de los clusters a las CGV arrojan luz sobre los desafíos que encuentran las firmas nacionales en el plano internacional, su aproximación subestima la heterogeneidad del territorio en sus dimensiones sociopolíticas.

A partir de reforzar la teoría de los sistemas de innovación con los postulados del estructuralismo latinoamericano, los autores llegan a la conclusión de que la proximidad territorial (entendida en términos físicos, económicos, simbólicos y sociopolíticos) es importante a la hora del aprendizaje y la construcción de capacidades tecnológicas y que, por tanto, en el contexto de globalización, los factores globales hegemonizan estos procesos locales necesarios para el desarrollo económico.

En conclusión, para los autores, no entender la complejidad inherente a la apropiación del conocimiento innovador conlleva a suponer que los canales de contactos internacionales son una fuente de construcción de capacidades tecnológicas. Por el contrario, los casos exitosos de inserción mundial están cimentados en una compleja red local e institucional basada en la acumulación nacional de conocimiento. En conclusión, para Pessoa Matos et al. (2015) el enfoque de las CGV sobrestima los intereses particulares de las empresas líderes globales en el desarrollo económico del país o en el fortalecimiento de sus proveedores locales.

\section{CONCLUSIONES}

El concepto CGV es parte central de la agenda actual de investigaciones sobre desarrollo, comercio e inversiones. El mismo ha sido utilizado en cientos de publicaciones a lo largo de casi 25 años para comprender y analizar distintos casos en la agricultura, la industria textil, la electrónica, los muebles, la energía, las aeronaves, la siderurgia, la industria farmacéutica, entre otros. 
Presenta importantes potencialidades, en especial al situar los análisis en el nivel de los actores sociales, para comprender las inversiones, a las empresas multinacionales y los procesos de desarrollo desde una óptica que privilegia la acción social por sobre las determinaciones estructurales.

A lo largo de este trabajo se han abordado las críticas realizadas al enfoque. En primer lugar, se expusieron los principales debates en torno a la forma particular en que se organiza la producción a nivel mundial. En este aspecto, si bien no existen miradas dicotómicas, diferentes autores resaltan distintas dimensiones a la hora de analizar quién y cómo se controlan las CGV. Se detectaron al menos tres variantes de cómo entender las cadenas, como conducción, como coordinación y como normatividad. Sturgeon (2008, p. 33) propone como síntesis que la gobernanza tienen tres pilares: las formas de manejar los activos específicos, la distribución del poder dentro de la cadena y el rol de las instituciones. En la intersección de estas tres dimensiones se pueden encontrar las formas específicas en que se gobiernan las cadenas.

Por último, se pasó revista a las tres posturas académicas en torno a los efectos que tuvieron las CGV sobre el desarrollo económico. Por un lado, están las posturas de la OMC, la OCDE y el Banco Mundial que sostienen que las CGV generan efectos positivos sobre el crecimiento, en la medida que se profundicen la apertura comercial como garantía de un adecuado aprovechamiento de las oportunidades que las mismas generan. Por otro lado, otra tendencia ligada a posturas neoschumpeterianas, sostiene que, si bien las cadenas establecen condiciones para el crecimiento, las mismas dependen de políticas activas en el upgrading de manera tal de que las empresas escalen hacia segmentos que agreguen más valor. Por último, la literatura ligada a los sistemas de innovación tiene una visión menos optimista sobre los impactos positivos de las CGV, al resaltar que los procesos de aprendizaje de las capacidades innovativas y tecnológicas depende de condiciones territoriales complejas y por tanto no aprehensibles de manera simple.

En síntesis, todavía persisten tensiones irresueltas en la academia en torno al rol de las cadenas globales en el desarrollo económico. Por un lado, los organismos internacionales depositan una fuerte confianza en las políticas comerciales y fiscales, los neoschumpeterianos en las políticas industriales o sectoriales, mientras que el último grupo analizado focaliza en las políticas de innovación. Tal vez, una mayor cantidad de análisis empíricos de caso puedan terminar de dirimir estas diferencias.

Ahora bien, en toda la literatura revisada existe una dimensión no estudiada sobre el sector empresarial. Los análisis sobre la convergencia entre las formas de distribución de poder dentro de la cadena (gobernanza) y las posibilidades de 
desarrollo (upgrading) dan por sentado la voluntad concreta de los actores de las cadenas de avanzar hacia eslabones de mayor valor agregado. Esta sobrestimación deposita en las empresas líderes, o incluso en los proveedores, una capacidad inmanente de ser actores capaces de emprender el desarrollo económico de un país. Bajo estas propuestas ${ }^{5}$ basta con que existan algunas condiciones determinadas y una cierta combinación específica de políticas económicas e institucionales para que se active la voluntad de ascender en las cadenas globales que toda empresa porta. En consecuencia, se asume que los actores sociales, sean empresas líderes o proveedores, buscan trepar hacia mejores eslabones. Por tanto, se vuelve necesario dar cuenta de que estos actores se encuentran enraizados de manera social y económica en estas situaciones de subdesarrollo, que en las mismas logran reproducir esquemas productivos beneficiosos y que entonces pueden no existir incentivos para transformar dicha realidad. Sería relevante para futuras líneas de investigación la profundización en una teoría de la acción empresaria en el marco de las CGV, que logre dar cuenta de actores sociales con orientaciones que pueden ser contradictorias, incluso paradojales, con una racionalidad económica de carácter muchas veces limitada, que no siempre son capaces de reconocer sus propios intereses o que incluso los mismos pueden encontrarse en oposición al desarrollo económico del país donde alojan sus actividades. Esta futura agenda investigativa podrá dar el marco teórico a nuevos trabajos empíricos, que analicen tanto las políticas estatales de upgrading como las respuestas empresariales a los desafíos de retener actividades que agreguen más valor en las cadenas globales.

\section{BIBLIOGRAFÍA}

Cardeillac, Joaquín. (2013). Sobre Cadenas Globales de Valor. Documento de trabajo n. ${ }^{\circ} 88$. Montevideo: Universidad de la República, 14 p.

Dalle, Demián; Fossati, Verónica y Lavopa, Federico. (2013). Política industrial: ¿el eslabón perdido en el debate de las Cadenas Globales de Valor? En: Revista Argentina de Economía Internacional, n.o 2, p. 3-16.

Dussel, Enrique (Coord.). (2018). Cadenas Globales de Valor. Metodología, teoría y debates. Ciudad de México: Universidad Nacional Autónoma de México, 228 p.

Feenstra, Robert. (1998). Integration of Trade and Disintegration of Production in the Global Economy. En: The Journal of Economic Perspectives, vol. 12, n. ${ }^{\circ} 4$, p. 31-50.

Gereffi, Gary. (1994). The Organization of Buyer-Driven Global Commodity Chains: How U.S. Retailers Shape Overseas Production Networks. En: Gereffi, Gary y Korzeniewicz, Miguel (Eds.). Commodity Chains and Global Capitalism. California: Praeger, p. 95-122.

5 Las versiones más pesimistas de las propuestas ancladas en los sistemas de innovación retoman más concretamente esta crítica. 
Gereffi, Gary; Humphrey, John y Sturgeon, Timothy. (2005). The governance of global value chains. En: Review of International Political Economy, vol. 12, n. ${ }^{\circ}$ 1, p. 78-104. Doi: 10.1080/09692290500049805

Gereffi, Gary y Sturgeon, Timothy. (2013). Global Value Chain-Oriented Industrial Policy: The Role of Emerging Economies. En: Elms, Deborah y Low, Patrick (Eds.).Global Value Chains in a Changing World. Ginebra: World Trade Organization, p. 329-360.

Gereffi, Gary. (2018). Políticas de desarrollo productivo y escalamiento: la necesidad de vincular empresas, agrupamientos y cadenas de valor. En: Dussel, Peter (comp.), Cadenas globales de valor: metodología, teoría y debates. Ciudad de México: Universidad Nacional Autónoma de México.

Gibbon, Peter; Bair, Jennifer y Ponte, Stefano. (2008). Governing Global Value Chains: An Introduction. En: Economy and Society, vol. 37, n. 3 , p. 315-338. Doi: doi. org/10.1080/03085140802172656.

Granovetter, Mark. (1985). Economic Action and Social Structure: The Problem of Embeddedness. En: American Journal of Sociology, vol. 91, n.o 3, p. 481-510.

Hopkins, Terence y Wallerstein, Immanuel. (1986). Commodity Chains in the World-Economy Prior to 1800. En: Review (Fernand Braudel Center), vol. 10, n.o 1, p. 157-170.

Humphrey, John y Schmitz, Hubert. (2002). How Does Insertion in Global Value Chains Affect Upgrading in Industrial Clusters? En: Regional Studies, vol. 36, n.o 9, p. 1017-1027. Doi: doi. org/10.1080/0034340022000022198.

Milberg, William y Winkler, Deborah. (2013). Outsourcing Economics: Global Value Chains in Capitalist Development. Cambridge: Cambridge University Press, 376 p.

OMC -Organización Mundial del Comercio-; OCDE -Organización para la Cooperación y el Desarrollo Económico-y Banco Mundial. (2014). Global Value Chains: Challenges, Opportunities and Implications for Policy. G20 Trade Ministers Meeting. Sydney, 53 p.

OMC -Organización Mundial del Comercio-; OCDE -Organización para la Cooperación y el Desarrollo Económico- y Banco Mundial. (2013). Implications of Globlal Value Chains for Trade, Investment, Development and Jobs. G-20 Leaders Summit. San Petersburgo, 29 p.

Pietrobelli, Carlo y Rabellotti, Roberta. (2011). Global Value Chains Meet Innovation Systems: Are There Learning Opportunities for Developing Countries? En: World Development, vol. 39, n.o 7, p. 1261-1269. Doi: doi.org/10.1016/j.worlddev.2010.05.013

Pessoa Matos, Marcelo; Cassiolato, José, Marcellino, I. y Podcameni, María. (2015). The myth of upgrading and development through insertion in Global Value Chains: a critique based on the Innovation System Literature. 13th Globelics Internatinal Conference. La Habana, 31 p.

Ponte, Stefano y Gibbon, Peter. (2005). Quality Standards, Conventions and the Governance of Global Value Chains. En: Economy and Society, vol. 34, n. 1, p. 1-31. Doi: doi. org/10.1080/0308514042000329315

Porta, Fernando; Santarcángelo, Juan y Schteingart, Daniel. (2017). Cadenas globales de valor y desarrollo económico. En: Economía y Desafíos del Desarrollo, vol. 1, n. ํ 1, p. 28-46. 
Santarcángelo, Juan; Schteingart, Daniel y Porta, Fernando. (2017). Cadenas globales de valor: una mirada crítica a una nueva forma de pensar el desarrollo. En: Cuadernos de Economía Crítica, n. ${ }^{\circ}$ 7, p. 99-129.

Sturgeon, Timothy. (2008). From Commodity Chains to Value Chains: Interdisciplinary Theory Building in an Age of Globalization. Massachusetts: Massachusetts Institute of Technology, $39 \mathrm{p}$.

Velázquez, Marisol y Tenorio, Alejandra. (2017). Cadenas globales de valor: una propuesta metodológica para el análisis de encadenamientos cafetaleros en México. En: Perspectivas Rurales. Nueva Época, vol. 15, n.o 29, p. 13-41. 